\title{
Effect of Maxwell stresses on the thermal crack tip field for piezoelectric
}

\section{materials}

\author{
A.B. Zhang $^{1 *}$, B.L. Wang ${ }^{1,2}$ \\ ${ }^{1}$ Graduate School at Shenzhen, Harbin Institute of Technology, Harbin 150001, P.R. China \\ ${ }^{2}$ Institute for Infrastructure Engineering, University of Western Sydney, Penrith, NSW 2751, \\ Australia
}

\begin{abstract}
Influence of Maxwell stresses acting upon crack faces on the fracture mechanics problem in piezoelectric materials subjected to thermal, mechanical and electric loads at infinity is investigated theoretically. The thermoelectric semi-permeable crack boundary condition is used. Explicit and closed-form solutions of thermal flux and electric displacement inside the crack, and stress and electric displacement intensity factors are derived based on the Stroh's formalism and thermoelectroelastic Green's function method. Numerical results are given to illustrate the effect of Maxwell stresses on the thermal stress intensity factor when the interior of the crack is filled with different dielectric medium.
\end{abstract}

Keywords: Piezoelectric; Maxwell stresses; Thermal stress; Crack

\section{Introduction}

Piezoelectric materials have been extensively used in engineering structures as sensors, actuators and transducers due to their pronounced piezoelectric, dielectric and pyroelectric properties. The analysis of piezoelectric solids weakened by cracks has drawn much attention of researchers because of the susceptibility of these materials to cracking. Concern for structural reliability and durability necessitates a better

\footnotetext{
${ }^{*}$ Corresponding author. Tel.: +86 75526032119

E-mail address: aibingzhang.hit@gmail.com (A.B. Zhang).
} 
understanding of the damage and fracture process of piezoelectric materials subjected to mechanical, thermal and electric loads. The fracture mechanics of these materials have attracted many theoretical studies, see [1-9].

Electrostatic forces, which initiate form the electric field between a solid and vacuum, air or any other insulating matter due to the difference in dielectric constants, behave as the external tractions. Forces and related displacements are induced at the crack surfaces of piezoelectric materials. This electrostatic forces is generally known as "Maxwell stresses" in the continuum mechanics theory. The electrostatic tractions acting upon crack faces should be introduced in piezoelectric materials fracture evaluation together with the semi-permeable crack model, especially when the mechanical load is not too large and the electric displacement is not too small [10]. The problem of Maxwell stresses and stored energy for deformable dielectric solids was discussed by McMeeking and Landis [11]. A nonlinear field theory of deformable dielectrics was presented by Suo et al. [12]. The influence of electrostatic tractions on the fracture behavior of an infinite piezoelectric material under mechanical and/or electric loads was investigated by Ricoeur and Kuna [13], and Xie et al. [14], respectively. Wang [15] studied that the effect of the Maxwell stresses on the interface crack between two dissimilar piezoelectric materials.

Taking Maxwell stresses into consideration, this paper investigates the crack problem in a piezoelectric material under the thermal, mechanical and electric loads. In section 2 the basic equations are outlined. In section 3 thermopiezoelectric fracture mechanics problem is studied based on the thermally and electrically semi-permeable crack model. Some numerical results are presented in section 4 , and concluding remarks are given in section 5. 


\section{Basic equations}

Consider a linear piezoelectric material in which all fields are assumed to depend only on the in-plane coordinates $x_{1}$ and $x_{2}$, and the poling direction is along the $x_{2}$-axis. The stress and electric displacement tensor $\mathbf{t}$, the elastic displacement and electric potential vector $\mathbf{U}$ and the temperature $T$ in the material subjected to external loads can be expressed in terms of complex analytic functions as follows [16, 17]:

$$
\begin{aligned}
& \mathbf{U}=\operatorname{Im}\left[\mathbf{A f}(z)+\mathbf{c} g\left(z_{t}\right)\right] \\
& \boldsymbol{\Phi}=\operatorname{Im}\left[\mathbf{B f}(z)+\mathbf{d} g\left(z_{t}\right)\right] \\
& \mathbf{t}_{1}=-\boldsymbol{\Phi}_{, 2}, \mathbf{t}_{2}=\boldsymbol{\Phi}_{, 1} \\
& T=\operatorname{Im}\left[g^{\prime}\left(z_{t}\right)\right]
\end{aligned}
$$

where a comma (or a prime) followed by an argument represents the differentiation with respect to the argument, "Im" stands for the imaginary part of a complex number, $\mathbf{U}=\left[u_{1} u_{2} u_{3} \varphi\right]^{T}, \quad \mathbf{t}_{j}=\left[\sigma_{1 j} \sigma_{2 j} \sigma_{3 j} D_{j}\right]^{T}, \quad j=1,2 ; \mathbf{f}(z)=\left[f_{1}\left(z_{1}\right) f_{2}\left(z_{2}\right) f_{3}\left(z_{3}\right) f_{4}\left(z_{4}\right)\right]^{T}, \mathbf{f} \quad$ and

$g$ are functions of the generalized complex variables $z_{i}$ and $z_{t}$, defined by $z_{i}=x_{1}+p_{i} x_{2}$ and $z_{t}=x_{1}+p_{*} x_{2}$, where $p_{i}$ and $p_{*}$ depend on the material constants. A, B, $\mathbf{c}$ and $\mathbf{d}$ are well-defined in the literature [17].

The Maxwell stresses acting on the crack faces in electrically and mechanically loaded piezoelectric material are [13]

$$
\sigma_{22}^{M}=\frac{1}{2} \frac{\left(D_{2}^{c}\right)^{2}}{\kappa_{c}}\left(1-\frac{\kappa_{c}}{\kappa_{m}}\right)
$$

where $\kappa_{c}$ is the permittivity of air, vacuum or others dielectric medium filling the crack, and $\kappa_{m}$ is the dielectric constant of piezoelectric material. $D_{2}^{c}$ is the electric displacement inside the crack to be determined, calculated form the capacitor analogy model [18] 


$$
D_{2}^{c}=-\kappa_{c} \frac{\varphi^{+}-\varphi^{-}}{u_{2}^{+}-u_{2}^{-}}
$$

The superscript "+" and "--" refer to the upper and lower main crack surfaces, respectively.

In order to consider the thermal effect of the medium inside the crack, it is more realistic to adopt the opening crack model developed by Ricoeur and Kuna [19]

$$
h_{2}^{c}=-\lambda_{c} \frac{T^{+}-T^{-}}{u_{2}^{+}-u_{2}^{-}}
$$

In Eq. (4), $h_{2}^{c}$ denotes the thermal flux normal to the crack faces, and $\lambda_{c}$ stands for the thermal conductivity of the medium inside the crack.

\section{Theoretical analysis}

Consider an infinite thermopiezoelectric medium problem of a single crack of length of $2 a$, the remote loading system is composed of thermal flux $h_{2}^{\infty}$, stresses $\sigma_{21}^{\infty}$, $\sigma_{22}^{\infty}$ and $\sigma_{23}^{\infty}$, and electric displacement $D_{2}^{\infty}$, as shown in Fig. 1. By the superposition principle, the crack face boundary conditions can be stated as follows

$$
\begin{array}{ll}
h_{2}=-\left(\hbar_{2}-q\right), & || x_{1}<, a \quad \underline{x} \neq \\
\mathbf{t}_{2}=\boldsymbol{t}^{c}{ }_{2} & |x|_{1}<a, x_{2}=1
\end{array}
$$

where $\mathbf{t}_{2}^{c}=\left[\sigma_{21}^{\infty}, \sigma_{22}^{\infty}-\sigma_{22}^{M}, \sigma_{23}^{\infty}, D_{2}^{\infty}-D_{2}^{c},\right]$.

The crack problem can be formulated in terms of thermoelectroelastic Green's function derived by Zhang and Wang [20]. Thermal flux boundary conditions, given by the first of Eq. (5), can be satisfied by distributing Green's function with weight $\theta(\xi)$ in the interval $\left|x_{1}\right|<a$ as

$$
-K \int_{-a}^{a} \frac{\theta(\xi)}{x_{1}-\xi} d \xi=-\left(h_{2}^{\infty}-h_{2}^{c}\right)
$$

where $K=-\lambda_{33}\left(p_{*}-\overline{p_{*}}\right) /(4 \pi i)$ is a real constant, and the auxiliary condition 


$$
\int_{-a}^{a} \theta(\xi) d \xi=0
$$

Singular integral Eq. (6), combined with Eq. (7), have the solution

$$
\theta(\xi)=\frac{h_{2}^{c}-h_{2}^{\infty}}{\pi K} \frac{\xi}{\sqrt{a^{2}-\xi^{2}}}
$$

The temperature jump across the crack can be obtained as

$$
\Delta T=\int_{x_{1}}^{a} \theta(\xi) d \xi=\frac{h_{2}^{c}-h_{2}^{\infty}}{\pi K} \sqrt{a^{2}-x_{1}^{2}}
$$

Then, the stress and electric displacement field on the crack faces may be found in the following form,

$$
\mathbf{t}_{2}=\left[\left(h_{2}^{\infty}-h_{2}^{c}\right) / K\right] \mathbf{G} x_{1}, \quad\left|x_{1}\right|<a
$$

where $\mathbf{G}=\frac{1}{4 \pi i}\left\{\mathbf{d}-\overline{\mathbf{d}}+\left[\left(\overline{\mathbf{B B}^{T}}-\mathbf{B B}^{T}\right)(\mathbf{c}+\overline{\mathbf{c}})+\left(\overline{\mathbf{B A}^{T}}-\mathbf{B A}^{T}\right)(\mathbf{d}+\overline{\mathbf{d}})\right]\right\}$ is the material property matrix.

To satisfy the stress and electric displacement boundary conditions Eq. $(5)_{2}$, the following singular integral equation is derived based on the thermoelectroelastic Green's function method

$$
\int_{-a}^{a} \frac{\mathbf{d}(\xi)}{\pi\left(x_{1}-\xi\right)} d \xi+\frac{h_{2}^{\infty}-h_{2}^{c}}{K} \mathbf{G} x_{1}+\mathbf{t}_{2}^{c}=0,\left|x_{1}\right|<a
$$

where $\mathbf{Y}=\mathbf{L}+\overline{\mathbf{L}}, \quad \mathbf{L}=i \mathbf{A} \mathbf{B}^{-1}, \quad \mathbf{b}=\mathbf{Y d}$ and $\mathbf{b}=\left[b_{1}, b_{2}, b_{3}, b_{4}\right]^{T}$ is the Burgers vector. The single value condition of the displacement and electric potential around a closed contour surrounding the whole crack requires that

$$
\int_{-a}^{a} \mathbf{d}(\xi) d \xi=0,\left|x_{1}\right|<a
$$

The solution of Eqs. (11) and (12) is given by

$$
\mathbf{d}(\xi)=\frac{h_{2}^{\infty}-h_{2}^{c}}{2 K} \mathbf{G} \frac{2 \xi^{2}-a^{2}}{\sqrt{a^{2}-\xi^{2}}}+\mathbf{t}_{2}^{c} \frac{\xi}{\sqrt{a^{2}-\xi^{2}}}
$$


Then, the displacement and electric potential jump along the crack surface can be expressed as

$$
\Delta \mathbf{U}=\int_{x_{1}}^{a} \mathbf{b}(\xi) d \xi=\frac{h_{2}^{\infty}-h_{2}^{c}}{2 K} \mathbf{Y G} x_{1} \sqrt{a^{2}-x_{1}^{2}}+\mathbf{Y t}_{2}^{c} \sqrt{a^{2}-x_{1}^{2}}
$$

According to Eqs. (3) and (15) the equation of electric displacement $D_{2}^{c}$ on the crack surfaces can be determined as

$$
D_{2}^{c}=-\kappa_{c} \frac{Y_{42}\left(\sigma_{22}^{\infty}-\sigma_{22}^{M}\right)+Y_{44}\left(D_{2}^{\infty}-D_{2}^{c}\right)}{Y_{22}\left(\sigma_{22}^{\infty}-\sigma_{22}^{M}\right)+Y_{24}\left(D_{2}^{\infty}-D_{2}^{c}\right)}
$$

Eq. (16) can be reduced to:

$$
\alpha_{3}\left(D_{2}^{c}\right)^{3}+\alpha_{2}\left(D_{2}^{c}\right)^{2}+\alpha_{1} D_{2}^{c}+\alpha_{0}=0
$$

with

$$
\begin{aligned}
& \alpha_{3}=\frac{Y_{22}}{2 \kappa_{c}}\left(1-\frac{\kappa_{c}}{\kappa_{m}}\right), \alpha_{2}=Y_{24}+\frac{Y_{42}}{2}\left(1-\frac{\kappa_{c}}{\kappa_{m}}\right), \alpha_{1}=-\left[Y_{22} \sigma_{22}^{\infty}+Y_{24} D_{2}^{\infty}-\kappa_{c} Y_{44}\right], \\
& \alpha_{0}=-\kappa_{c}\left[Y_{42} \sigma_{22}^{\infty}+Y_{44} D_{2}^{\infty}\right] .
\end{aligned}
$$

Eq. (16) is a cubic equation and its general solution can be found from mathematics handbook. For reasonable material constants and loading conditions, Eq. (16) has three roots, but only one root is physically acceptable. In this paper, the solution has the following form:

$$
D_{2}^{c}=-\frac{\alpha_{2}}{3 \alpha_{3}}+\frac{-1-\sqrt{3} i}{2} \sqrt[3]{P+\sqrt{P^{2}+Q^{3}}}+\frac{-1+\sqrt{3} i}{2} \sqrt[3]{P-\sqrt{P^{2}+Q^{3}}}
$$

where $P$ and $Q$ are two known real constants depending on the applied loads, the material constants and the permittivity of the dielectric medium inside the crack as formulated below:

$$
P=\frac{\alpha_{2} \alpha_{1}}{6 \alpha_{3}^{2}}-\frac{\alpha_{2}^{3}}{27 \alpha_{3}^{3}}-\frac{\alpha_{0}}{2 \alpha_{3}}, \quad Q=\frac{\alpha_{1}}{3 \alpha_{3}}-\frac{\alpha_{2}^{2}}{9 \alpha_{3}^{2}}
$$

From Eqs. (4), (9) and (14), it follows that the thermal flux inside the crack is 


$$
h_{2}^{c}=\frac{\lambda_{c} h_{2}^{\infty}}{\pi K\left[Y_{22}\left(\sigma_{22}^{\infty}-\sigma_{22}^{c}\right)+Y_{24}\left(D_{2}^{\infty}-D_{2}^{c}\right)\right]+\lambda_{c}}
$$

The stress fields at the right crack tip on the plane $x_{2}=0$ are obtained by Eqs. (11) and (13)

$$
\mathbf{t}_{2}=\frac{h_{2}^{\infty}-h_{2}^{c}}{2 K} \mathbf{G} \frac{a^{2}}{\sqrt{x_{1}^{2}-a^{2}}}+\mathbf{t}_{2}^{c}\left[\frac{x_{1}}{\sqrt{x_{1}^{2}-a^{2}}}-1\right]
$$

Defining the field intensity factor vector

$$
\mathbf{K}=\left[K_{I I}, K_{I}, K_{I I I}, K_{D}\right]^{T}=\lim _{x_{1} \rightarrow a^{+}} \sqrt{2 \pi\left(x_{1}-a\right)} \mathbf{t}_{2}
$$

Then, from Eqs. (20) and (21), we obtain:

$$
\mathbf{K}=\frac{h_{2}^{\infty}-h_{2}^{c}}{2 K} \mathbf{G} \sqrt{\pi} a^{3 / 2}+\sqrt{\pi a} \mathbf{t}_{2}^{c}
$$

\section{Numerical results and discussion}

The influence of electrostatic tractions on the fracture behavior of piezoelectric materials was fully studied by Ricoeur and Kuna [13], and Zhang and Wang [21]. In this section, we only discuss the effect of Maxwell stresses acting upon crack faces on thermal stress intensity factor by using the numerical results. The material is subjected to a uniform thermal flux $h_{2}^{\infty}$, a uniform electric displacement $D_{2}^{\infty}$, and a uniform tensile stress $\sigma_{22}^{\infty}$ remote from the crack. The thermopiezoelectric material PZT-5A is adopted, and its properties are as follows [22]

Elastic constants:

$$
c_{11}=99.2 \mathrm{GPa}, c_{12}=54.02 \mathrm{GPa}, c_{13}=50.78 \mathrm{GPa}, c_{33}=86.86 \mathrm{GPa}, c_{44}=21.1 \mathrm{GPa}
$$

Piezoelectric constants:

$$
e_{31}=-7.21 \mathrm{C} / \mathrm{m}^{2}, e_{33}=15.12 \mathrm{C} / \mathrm{m}^{2}, e_{15}=12.32 \mathrm{C} / \mathrm{m}^{2}
$$

Dielectric constants: 
$\kappa_{11}=153 \times 10^{-10} \mathrm{~F} / \mathrm{m}, \kappa_{33}=150 \times 10^{-10} \mathrm{~F} / \mathrm{m}$

Heat conduction constants:

$\lambda_{11}=1.8 \mathrm{~W} / \mathrm{Km}, \lambda_{33}=1.8 \mathrm{~W} / \mathrm{Km}, \lambda_{13}=0$

Thermal-stress coefficient:

$\beta_{11}=0.3314 \times 10^{6} \mathrm{~N} / \mathrm{Km}^{2}, \beta_{33}=0.326 \times 10^{6} \mathrm{~N} / \mathrm{Km}^{2}$

Pyroelectric constants:

$\gamma_{3}=-8.61 \times 10^{-6} \mathrm{C} / \mathrm{Km}^{2}$

The thermal conductivity and electric permeability of air or vacuum are $\lambda_{0}=0.024 \mathrm{~W} / \mathrm{Km}$ and $\kappa_{0}=0.0885 \times 10^{-10} \mathrm{~N} / \mathrm{A}^{2}$, respectively. In Figs. 2 and 3 the normalized thermal stress intensity factors $K_{I I} / K_{I I 0}$ are plotted vs. the applied electric loads $D_{2}^{\infty}$ for different mechanical loads and dielectric constants inside of crack, $K_{I I 0}$ is the value of thermal stress intensity factor according to the thermally impermeable crack assumption. It can be found that $K_{I I} / K_{I I 0}$ varies only slightly with the applied electric displacement for $\kappa_{c} / \kappa_{0}=10,100,1000$; but for $\kappa_{c} / \kappa_{0}=1, K_{I I} / K_{I I 0}$ obviously increases markedly as $D_{2}^{\infty}$ is increased for a given mechanical loads. That is, the effect of Maxwell stresses on the thermopiezoelectric material fracture mechanics evaluation should be considered when the medium inside the crack is full of air or vacuum. It is clear that thermal stress intensity factors obtained are smaller than that without considering the crack faces electrostatic tractions. Fig. 4, combined with Figs. 2 and 3, show that $K_{I I} / K_{I I 0}$ increases as the applied mechanical load $\sigma_{22}^{\infty}$ increases for a given $D_{2}^{\infty}$ and $\kappa_{c}$. It is also found that the effect of Maxwell stresses at the crack surfaces on thermal stress intensity factor may be neglected for large mechanical loads and limited electric field as well as large dielectric constants of the crack interior. 
Normalized thermal stress intensity factor $K_{I I} / K_{I I 0}$ vs. thermal conductivity of crack interior are plotted for the case of $\sigma_{22}^{\infty}=10 \mathrm{MPa}$ and $D_{2}^{\infty}=0.01 \mathrm{Cm}^{-2}$ in Figs. 5 and 6. It can be found that when $\lambda_{c}$ rising, $K_{I I} / K_{I I 0}$ is decreasing rapidly. Further, Taking the Maxwell stresses effect into consideration, Fig. 6 shows that the value of $K_{I I} / K_{I I 0}$ obtained is smaller than that without considering it. It is attributed to the fact that the electrostatic crack surface stress influences on the crack opening displacement, then on the thermal flux inside the crack, further on the thermal stress intensity factor.

\section{Conclusions}

In the present paper, thermoelectric medium crack model is applied to simulate the fracture mechanics problem in a piezoelectric solid by considering the Maxwell stresses effect. The thermal flux and electric displacement inside the crack are assumed to be dependent on the crack opening displacement. A system of singular integral equations is derived to model the crack problem based on the Stroh's formulation and the thermoelectroelastic Green's function method. Expressions for stress and electric displacement intensity factors at the crack tip are given in closed-form. Numerical results show that the influence of Maxwell stresses acting upon crack surfaces on the thermal stress intensity factor may be neglected for large dielectric constants of the crack interior and large mechanical loads, as well as small electrical loads.

\section{Acknowledgements}

The research was supported by the National Science Foundation of China (NSFC) (Project Nos. 11172081 and 11372086), and China Postdoctoral Science Foundation 
(Project No. 2014M550185). BLW was also supported by the Australian Research Council Future Fellowship program (Grant No. FT100100211).

\section{References}

[1] V.Z. Parton, Fracture mechanics of piezoelectric materials. Acta. Astronaut 3 (1976) 671-683.

[2] Y.E. Pak, Crack extension force in a piezoelectric material. J. Appl. Mech. 57 (1990) 647-653.

[3] Z. Suo, C.M. Kuo, D.M. Barnett, J.R. Willis, Fracture mechanics for piezoelectric ceramics. J. Mech. Phys. Solids 40 (1992) 739-765.

[4] H.J. Gao, T.Y. Zhang, T. Pin, Local and global energy release rates for an electrically yielded crack in a piezoelectric ceramic. J. Mech. Phys. Solids 45 (1997) 491-510.

[5] B.L. Wang, Y.W. Mai, On the electrical boundary conditions on the crack surfaces in piezoelectric ceramics. Int. J. Eng. Sci. 41 (2003) 633-652.

[6] T.Y. Zhang, C.F. Gao, Fracture behaviors of piezoelectric materials. Theor. Appl. Fract. Mech. 41 (2004) 339-379.

[7] V. Loboda, Y. Lapusta, A. Sheveleva, Electro-mechanical pre-fracture zones for an electrically permeable interface crack in a piezoelectric bimaterial. Int. J. Solids Struct. 44 (2007) 5538-5553.

[8] X.C. Zhong, K.S. Zhang, An opening crack model for thermopiezoelectric solids. Eur. J. Mech. A-Solids 41 (2013) 101-110.

[9] J. Sladek, V. Sladek, E. Pan, M. Wünsche, Fracture analysis in piezoelectric semiconductors under a thermal load. Eng. Fract. Mech. 126 (2014) 27-39.

[10] Q. Li, Y.H. Chen, Why traction-free? Piezoelectric crack and Coulombic traction. Arch. Appl. Mech. 78 (2008) 559-573. 
[11] R.M. McMeeking, C.M. Landis, Electrostatic forces and stored energy for deformable dielectric materials. J. Appl. Mech. 72 (2005) 581-590.

[12] Z.G. Suo, X.H. Zhao, W.H. Greene, A nonlinear field theory of deformable dielectrics. J. Mech. Phys. Solids 56 (2008) 467-486.

[13] A. Ricoeur, M. Kuna, Electrostatic tractions at crack faces and their influence on the fracture mechanics of piezoelectrics. Int. J. Fract. 157 (2009) 3-12.

[14] T. Xie, C.F. Fan, H.T. Liu, M.H. Zhao, T.Y. Zhang, Effect of electrostatic tractions on the fracture behavior of a piezoelectric material under mechanical and/or electric loading. Theor. Appl. Fract. Mech. 69 (2014) 6-16.

[15] Y.Z. Wang, Effects of Maxwell stress on interfacial crack between two dissimilar piezoelectric solids. J. Appl. Mech. 81(2014) 101003-1-101003-6.

[16] A.N. Stroh, Dislocations and cracks in anisotropic elasticity. Phil. Mag. 7 (1958) 625-646.

[17] Q.H. Qin, Y.W. Mai, Thermoelectroelastic Green's function and its application for bimaterial of piezoelectric materials. Arch. Appl. Mech. 68 (1998) 433-444.

[18] T.H. Hao, Z.Y. Shen, A new electric boundary condition of electric fracture mechanics and its applications. Eng. Fract. Mech. 47 (1994) 793-802.

[19] A. Ricoeur, M. Kuna, The thermoelectromechanical J-integral and the thermal permeability of cracks. Key Eng. Mater. 385-387 (2008) 569-572.

[20] A.B. Zhang, B.L. Wang, Contact zone approach for an electrically impermeable crack in piezoelectric materials under thermal-mechanical loading. J. Thermal Stresses 35 (2012.) 749-765.

[21] A.B. Zhang, B.L. Wang, The influence of Maxwell stresses on the fracture mechanics of piezoelectric materials. Mech. Mater. 68 (2014) 64-69.

[22] C.B. Liu, Z.G. Bian, W.Q. Chen, C.F. Lu, Three-dimensional pyroelectric 
analysis of a functionally graded piezoelectric hollow sphere. J. Thermal Stresses 35 (2012) 499-516. 


\section{List of figure captions}

Fig. 1 An opening crack model with the dielectric constants $\kappa_{c}$, and the thermal conductivity $\lambda_{c}$ of the medium inside the crack

Fig. 2 Variation of normalized thermal stress intensity factor with applied electric displacement, $\sigma_{22}^{\infty}=10 M P a$.

Fig. 3 Variation of normalized thermal stress intensity factor with applied electric displacement, $\sigma_{22}^{\infty}=20 M P a$.

Fig. 4 Variation of normalized thermal stress intensity factor with applied tensile loads, $D_{2}^{\infty}=0.01 \mathrm{Cm}^{-2}$.

Fig. 5 Normalized thermal stress intensity factor vs. thermal conductivity inside of crack, $\lambda_{c} / \lambda_{0}$ varies from 0 to 1 .

Fig. 6 Normalized thermal stress intensity factor vs. thermal conductivity inside of crack, $\lambda_{c} / \lambda_{0}$ varies from 0.01 to 0.1 . 


$$
\boldsymbol{\sigma}_{23}^{\infty} \underset{\leftrightarrow}{\boldsymbol{\sigma}_{22}^{\infty}} \boldsymbol{\sigma}_{21}^{\infty} \quad \boldsymbol{\Lambda} \boldsymbol{h}_{2}^{\infty}, \boldsymbol{D}_{2}^{\infty} \uparrow
$$

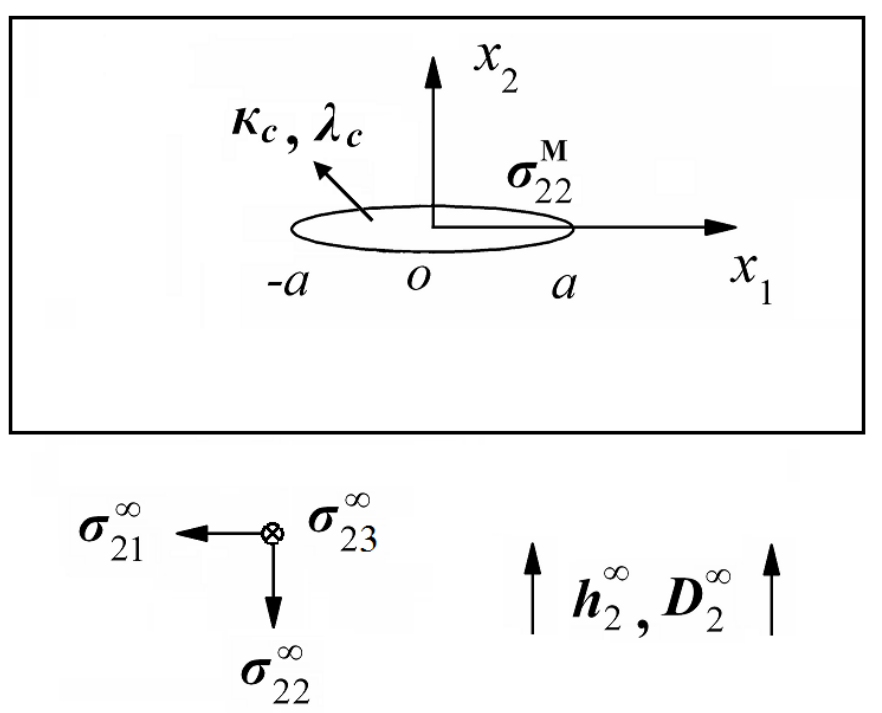

Fig. 1 


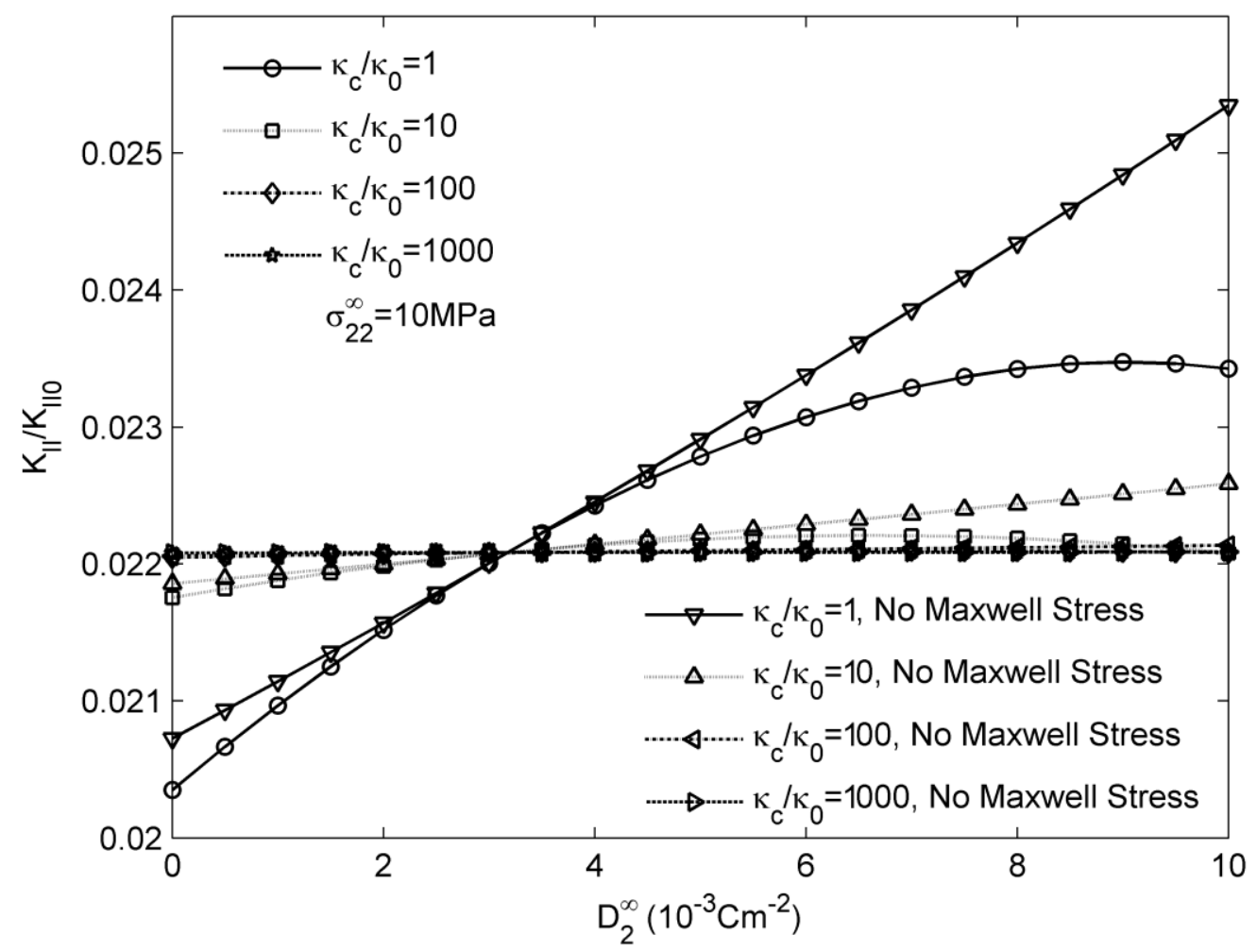

Fig. 2 


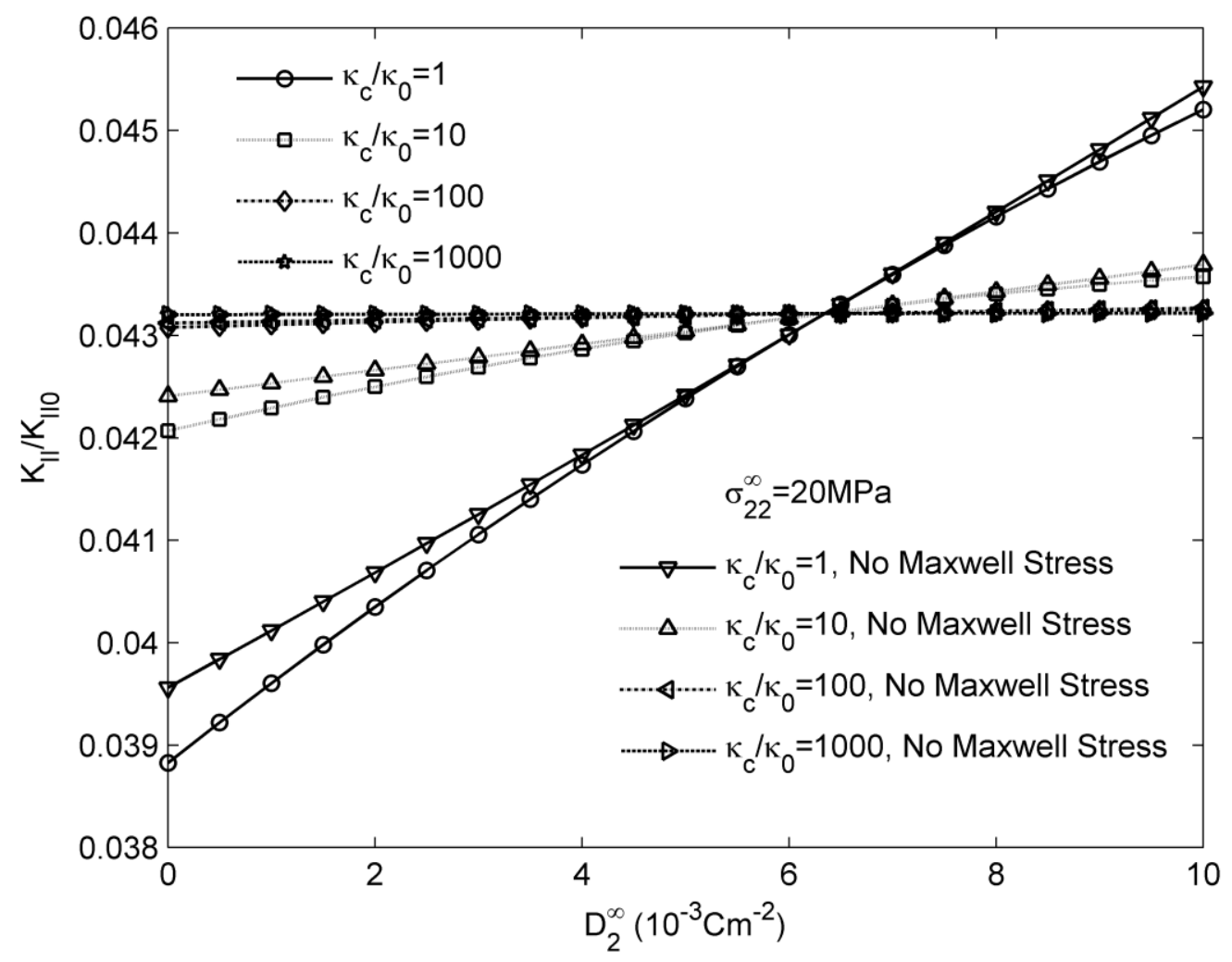

Fig. 3 


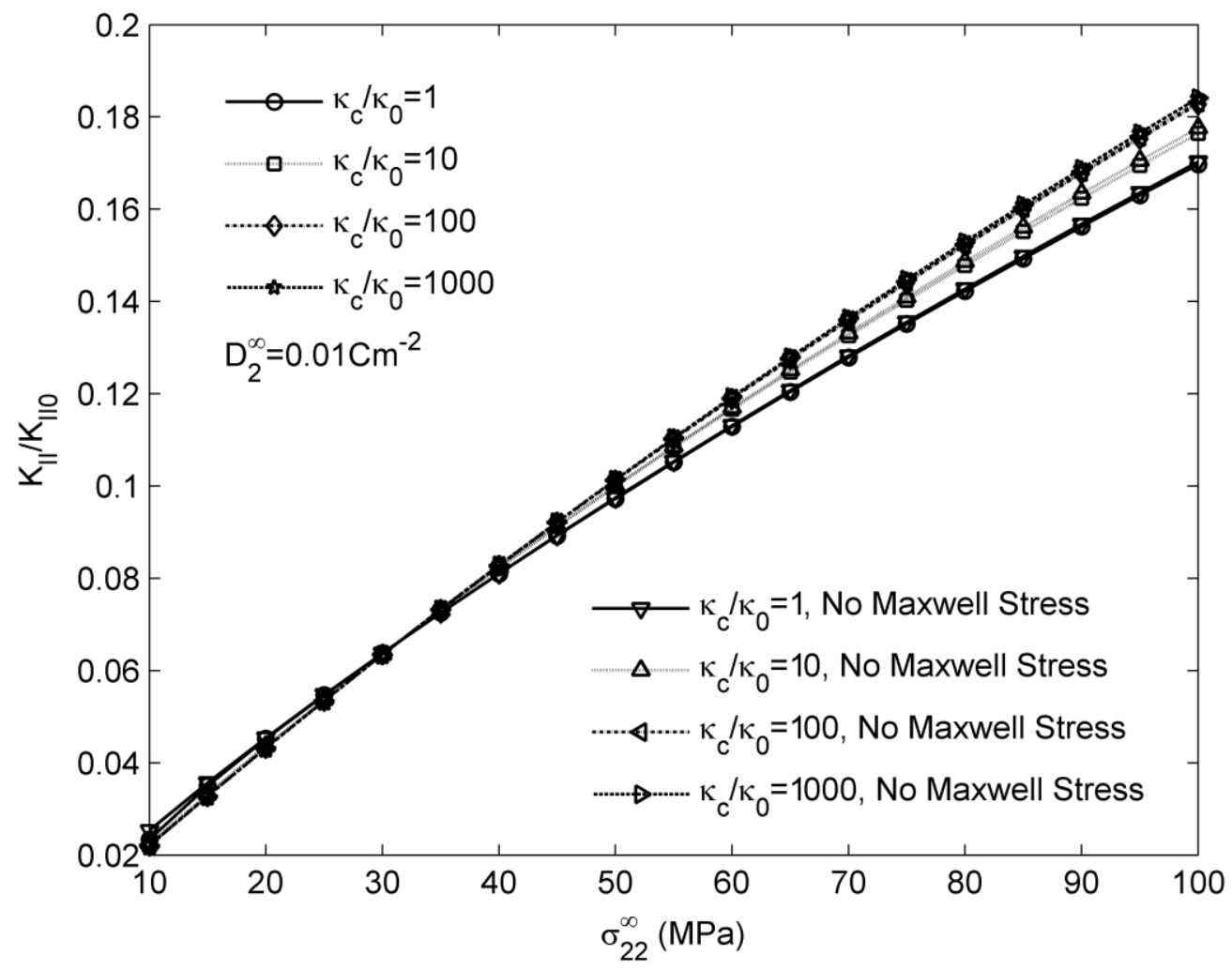

Fig. 4 


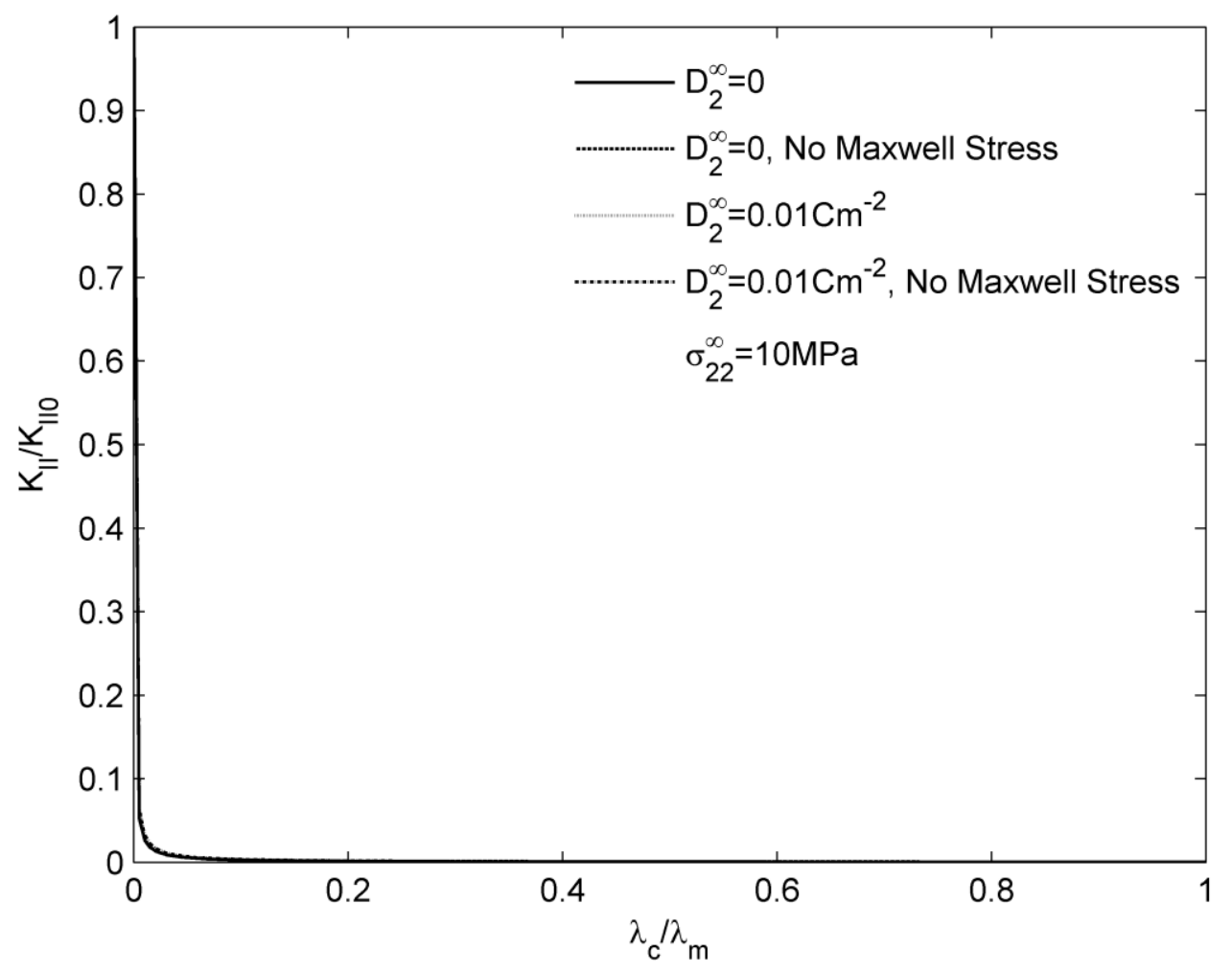

Fig. 5 


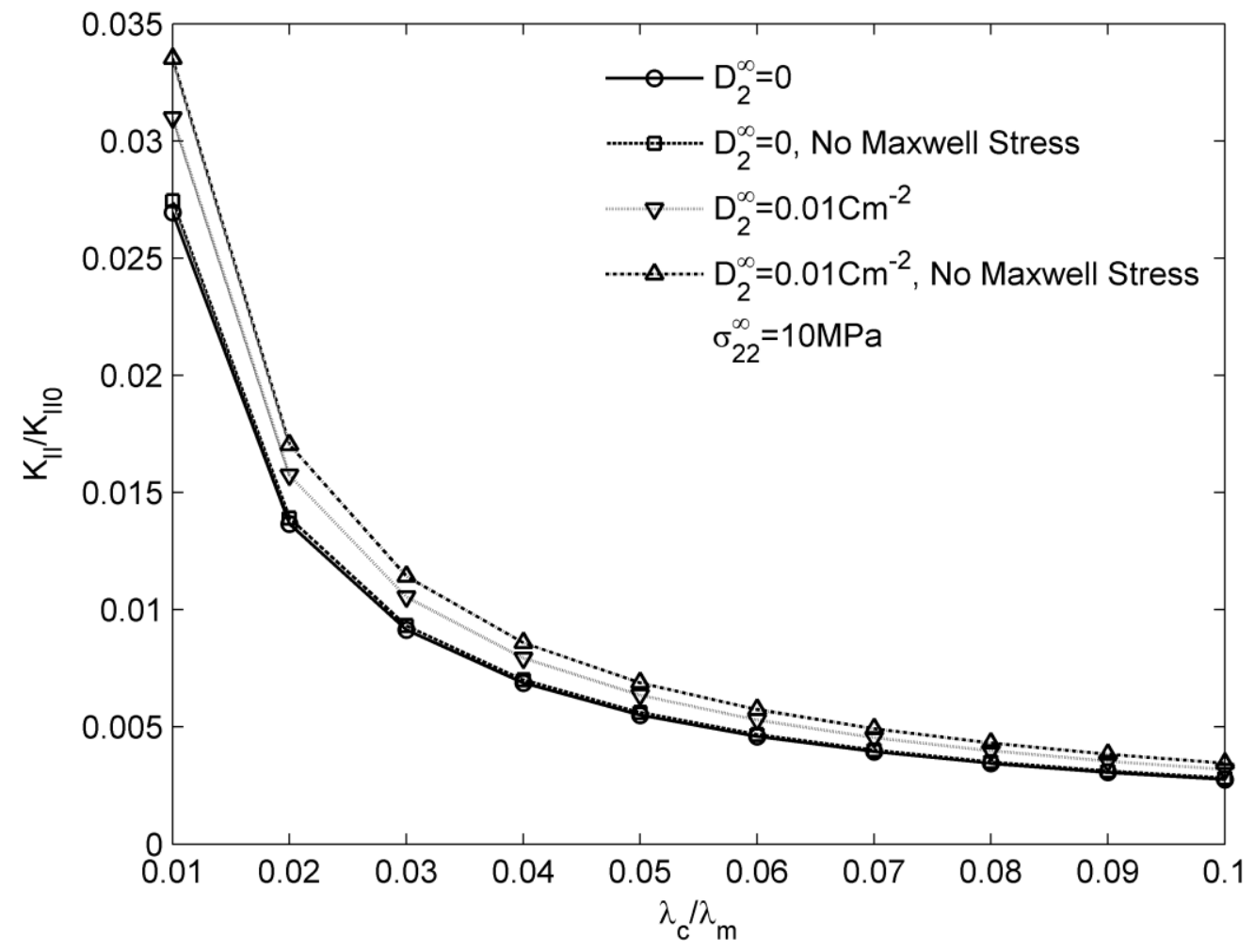

Fig. 6 